\title{
Virological Outcome After Choice of Antiretroviral Regimen Guided by Proviral HIV-1 DNA Genotyping in a Real-Life Cohort of HIV-Infected Patients
}

\author{
Agnès Meybeck, MD, Enagnon Kazali Alidjinou, MD, ${ }^{2}$ Thomas Huleux, MD, ${ }^{1}$ Anne Boucher, MD, ${ }^{1}$ \\ Macha Tetart, MD, ${ }^{1}$ Philippe Choisy, MS, Laurence Bocket, MD, ${ }^{1}$ Faiza Ajana, MD, and Olivier Robineau, MD ${ }^{1}$
}

\begin{abstract}
Issues have been raised concerning clinical relevance of HIV-1 proviral DNA genotypic resistance test (DNA GRT). To assess impact of DNA GRT on choice of antiretroviral therapy (ART) and subsequent virological outcome, we retrospectively reviewed decision-making and viral load (VL) evolution following DNA GRT performed in our center between January 2012 and December 2017, except those prescribed within the framework of a clinical trial. A total of 304 DNA GRTs were included, 185 (62\%) performed in a context of virological success. Only $34 \%$ of tests were followed by ART change, more frequently in situation of virological success $(39 \%$ vs. $26 \%, p=0.02)$. In this situation, ART change guided by DNA GRT led to VL $>20$ copies $/ \mathrm{mL}$ after 6 months in 5\% of cases. In multivariate analysis, higher HIV DNA quantification $(p=0.01)$ was associated with occurrence of viremia. A higher nadir of CD4 count $(p=0.04)$ and a longer time with VL $<20$ copies $/ \mathrm{mL}(p=0.04)$ were independently associated with a lower risk of viremia. In situation of low-level viremia, ART change guided by DNA GRT led to VL $<20$ copies $/ \mathrm{mL}$ after 6 months in $52 \%$ of cases, while decision to maintain the same treatment led to $\mathrm{VL}<20$ copies $/ \mathrm{mL}$ in $74 \%$ of cases. In multivariate analysis, longer time with $\mathrm{VL}>20$ copies $/ \mathrm{mL}(p=0.02)$ was associated with persistence of virological replication. In conclusion, in situation of virological success, use of DNA GRT in addition to analysis of historical RNA GRT to guide ART optimization appears safe. Its prescription framework in situation of low-level viremia deserves to be better defined.
\end{abstract}

Keywords: HIV-1, proviral DNA, genotypic resistance test, virological failure, virological suppression

\section{Introduction}

$\mathbf{F}$ OLLOWING EUROPEAN GUIDELINES, genotypic HIV drug resistance testing is recommended for persons with HIV infection at entry into care to guide selection of the initial antiretroviral therapy (ART) regimen and for antiretroviral therapy-experienced patients with virological failure to assist in the selection of active drugs when changing ART regimen. ${ }^{1}$ According to French guidelines, virological failure is defined by a viral load (VL) above 200 copies/mL. ${ }^{2}$ But ART-treated patients with low-level viremia have an increased probability of accumulation of additional drug resistance mutations. The number of fully active drugs and the duration of low-level viremia are predictive of emerging resistance. ${ }^{3}$

In patients with $\mathrm{VL}<500$ copies/mL, resistance testing may be unsuccessful. Genotypic resistance test (GRT) applied on proviral DNA extracted from peripheral blood mononuclear cells (PBMCs) could be an alternative. Genotypic information in HIV-1 DNA reflects patient history of virological failure during drug exposure. ${ }^{4-6}$ Archived mutations may be associated with subsequent treatment failure. ${ }^{7,8}$ Several studies confirmed a certain correlation between historical HIV-RNA resistance test results and proviral DNA genotyping in patients with suppressed or low-level viremia. 9,10

In addition to patients experimenting virological failure, patients with undetectable VL in whom a therapeutic switch is planned for simplification purposes or toxicity may also benefit from proviral DNA genotyping. Recent studies suggest an interest of proviral DNA GRT before switching therapy in patients successfully suppressed when no historical RNA resistance test is available. ${ }^{11,12}$ In France, November 2016 and May 2017 updates of national guidelines proposed

\footnotetext{
${ }^{1}$ Infectious Diseases Department, Tourcoing Hospital, Tourcoing, France.

${ }^{2}$ Virology Department, Lille University Hospital, Lille, France.

Parts of the data were presented at the 2018 Glasgow HIV Drug Therapy Congress.
} 
to realize proviral DNA GRT in PBMC in patients with virological failure with low-level viremia and unsuccessful RNA resistance test and in patients successfully suppressed when no historical RNA resistance test is available and a switch of therapy is planned. ${ }^{2}$

However, several issues have been raised concerning HIV proviral DNA GRT. It may not detect all mutations that have occurred over time. ${ }^{9,10}$ Consequently, positive test results may be more helpful and change relying on negative test results could expose to treatment failure. Conversely, clinical relevance of the detection of unexpected resistance to the current regimen in case of virological success is not clearly established. ${ }^{13}$ To assess impact of proviral DNA GRT in addition to analysis of historical RNA genotyping test on choice of ART and subsequent virological outcome, we retrospectively reviewed decision-making and VL evolution following the realization of each test performed in our center.

\section{Patients}

\section{Patients and hospital setting}

We performed a retrospective analysis of all HIV-1 proviral DNA GRT performed in our center, except those prescribed within the framework of a clinical trial, between January 2012 and December 2017. DNA GRTs performed in case of VL $>200$ copies/mL were also excluded from the analysis. One patient could benefit from more than one test during the study period.

\section{Data collection}

Demographic data and characteristics of all patients who benefited from DNA GRT were collected using clinical data base (Nadis). ${ }^{14}$ Following data on HIV infection were recorded: year of HIV diagnosis, nadir of CD4 cell count, zenith of HIV-VL, HIV disease staging according to CDC Classification System for HIV-Infected Adults (Category A: asymptomatic, acute HIV, or persistent generalized lymphadenopathy; Category B: symptomatic conditions not A or C; and Category C: AIDS-indicator conditions), the duration of undetectability, the number of RNA tests available, the time between the DNA test and the last and first RNA test available, proviral DNA quantification, CD4 cell count, and plasma HIV-VL at the time of DNA GRT performance.

Quantification of total HIV DNA level was performed in whole blood using a real-time PCR assay (Biocentric, Bandol, France) and results were expressed as copies $/ 10^{6}$ PBMCs. History of antiretroviral treatment was collected: number of successive antiretroviral regimens, duration of antiretroviral therapy, number of ART family with resistance, and current antiretroviral treatment at the time of DNA GRT performance.

\section{Resistance analysis}

The Agence Nationale de Recherche sur le SIDA genotypic algorithm was used to identify resistance-associated mutations. All except the fully susceptible HIV-1 sequences were considered as resistant in the analysis of the present study. Since for DNA provirus, impact of stop codons and G to A mutations on antiretroviral resistance is unknown, these mutations were counted in the rate of drug resistance mutations in the analysis. Results of DNA GRT were compared to those of historical HIV-RNA resistance tests. The rates of discordance between DNA GRT and historical RNA resistance tests were analyzed. Discordance occurred in two situations: resistance diagnosed by DNA GRT, but never detected in RNA or no RNA GRT available (DisDNA); or resistance absent from DNA GRT, but detected at least once in RNA (DisRNA).

\section{Antiretroviral therapy (ART) change and virological outcome}

In our study, virological failure was defined as persistent VL > 200 copies/mL. Low-level viremia was defined as persistent VL in range between lower limit of detection of assay (20 copies/mL) and 200 copies/mL. We distinguished DNA GRT performed in the context of virological success defined as VL $<20$ copies/mL from those performed in the context of low-level viremia, defined as VL between 20 and 200 copies/mL. ART changes made in the 6 months following DNA GRT were recorded.

Virological outcome was assessed by VL evolution in the 6 months following ART change or the DNA GRT in the absence of ART change. In case of DNA GRT performed in the context of virological success, occurrence of $\mathrm{VL}>20$ copies $/ \mathrm{mL}$ in the 6 months period was recorded. In case of DNA GRT performed in the context of low-level viremia, achievement of $\mathrm{VL}<20$ copies $/ \mathrm{mL}$ in the 6 months period was registered.

\section{Statistical analysis}

Continuous variables were expressed as mean and standard deviation. They were compared using the Mann-Whitney test. Categorical variables were expressed as number and percentage. They were compared using the Fisher's exact test. Differences between groups were considered to be significant for variables yielding a $p$ value $<0.05$.

In case of DNA GRT performed in the context of virological success, Kaplan-Meier analysis was used to estimate cumulative probability of maintenance of virological success. In case of DNA GRT performed in the context of lowlevel viremia, Kaplan-Meier analysis was used to estimate cumulative probability of persistence of virological failure. Log rank test was used to compare probability of virological success or failure in different groups (change vs. no change of antiviral treatment).

To determine the independent effect of the variables on virological outcome, we performed a logistic regression analysis using the purposeful selection of covariates. All covariates with $p<0.2$ in the unadjusted model were entered into the multivariate model. All statistical analyses were performed using Stata software version 7.0.

\section{Results}

\section{Study population}

From January 2012 to December 2017, a total of 343 HIV1 proviral DNA GRT were performed as routine laboratory monitoring. Thirty-nine DNA GRTs were excluded from the analysis: 11 tests prescribed while VL was higher than 1000 copies/mL, 23 prescribed while VL was between 200 and 1000 copies/mL, and 5 tests performed with no available concomitant HIV-VL. 
Table 1. Demographic Data and Characteristics of the Patients According to the Virological Status at the Time of DNA Genotypic Resistance Test Performance

\begin{tabular}{|c|c|c|c|c|}
\hline & Total $(\mathrm{n}=304)$ & $\begin{array}{c}V L>20 \text { copies } / m L \\
(\mathrm{n}=119)\end{array}$ & $\begin{array}{c}V L<20 \text { copies } / m L \\
(\mathrm{n}=185)\end{array}$ & $\mathrm{P}$ \\
\hline Age (years) & $48.7 \pm 11.4$ & $48.7 \pm 12.0$ & $48.6 \pm 11.0$ & 0.98 \\
\hline Male, $n(\%)$ & $220(72)$ & $90(76)$ & $130(70)$ & 0.37 \\
\hline CDC stage, $n(\%)$ & & & & 0.12 \\
\hline A & $142(47)$ & $50(42)$ & $92(49)$ & \\
\hline B & $53(17)$ & $18(15)$ & $35(19)$ & \\
\hline $\mathrm{C}$ & $109(36)$ & $51(43)$ & $58(31)$ & \\
\hline HIV type B & $178(59)$ & $76(61)$ & $102(55)$ & 0.4 \\
\hline Nadir CD4 count $\left(/ \mathrm{mm}^{3}\right)$ & $214 \pm 169$ & $187 \pm 149$ & $231 \pm 179$ & 0.03 \\
\hline Zenith VL (copies/mL) & $750,244 \pm 3,471,236$ & $851,001 \pm 1,409,378$ & $685,432 \pm 4,307,866$ & $<0.001$ \\
\hline CD4 count $\left(/ \mathrm{mm}^{3}\right)$ & $616 \pm 288$ & $521 \pm 226$ & $678 \pm 307$ & $<0.001$ \\
\hline VL (copies/mL) & & $72 \pm 43$ & & \\
\hline VL DNA (copies PBMC) & $1016 \pm 845$ & $1255 \pm 1095$ & $879 \pm 626$ & 0.016 \\
\hline $\begin{array}{l}\text { Time between HIV diagnosis and DNA } \\
\text { GRT (years) }\end{array}$ & $13.4 \pm 8.4$ & $12.1 \pm 8.5$ & $14.3 \pm 8.2$ & 0.002 \\
\hline $\begin{array}{l}\text { Time between last RNA GRT and DNA } \\
\text { GRT (years) }\end{array}$ & $4.7 \pm 4.2$ & $3.5 \pm 3.2$ & $6.2 \pm 4.5$ & $<0.026$ \\
\hline Number of previous RNA GRT & $2.2 \pm 2.4$ & $3.1 \pm 2.7$ & $1.5 \pm 2.0$ & $<0.001$ \\
\hline $\begin{array}{l}\text { Time between ART initiation and DNA } \\
\text { GRT (years) }\end{array}$ & $11.0 \pm 7.2$ & $9.9 \pm 7.3$ & $11.7 \pm 7.1$ & 0.029 \\
\hline $\begin{array}{l}\text { Time between last ART change and DNA } \\
\text { (years) }\end{array}$ & $3.4 \pm 2.3$ & $2.3 \pm 2.2$ & $4.1 \pm 3.3$ & $<0.001$ \\
\hline Number of previous ART regimens & $5.8 \pm 5.6$ & $6.4 \pm 6.3$ & $5.4 \pm 5.1$ & 0.31 \\
\hline
\end{tabular}

Mean (standard deviation) and number (\%).

ART, antiretroviral therapy; GRT, genotypic resistance test; PBMC, peripheral blood mononuclear cell; VL, viral load.

Significant results $(p<0.05)$ appear in bold.

Demographic data and characteristics of the patients according to the virological status at the time of DNA GRT performance are summarized in Table 1. Our patients were predominantly males $(72 \%)$, with a mean age of 49 years, and a long history of ART; the mean time between ART initiation and DNA GRT was $11 \pm 7.2$ years, with a mean of $5.8 \pm 5.6$ previous ART regimens. DNA GRT was performed in a context of a virological success of the current therapy in 185 cases $(62 \%)$. The two populations differed significantly as shown in Table 1.

\section{Resistance analysis}

No previous RNA GRT was available in 87 cases (29\%). Absence of historical RNA GRT was noted in a greater proportion of DNA GRT realized in a context of virological success $(38 \%$ vs. $14 \%, p<0.001)$. The virus was resistant according to historical RNA GRTs when available to a mean of 1.6 \pm 1.4 ART families. The rates of discordance between DNA GRT and historical RNA resistance tests concerning various antiretroviral drugs are summarized in Fig. 1. By comparison with historical HIV-RNA GRT results, 116 (38\%) DNA GRT did not detect a resistance previously known, but 88 (29\%) revealed at least one new resistance.

New resistances on DNA GRT concerned mainly rilpivirine with at least one resistance-associated mutation in $10.8 \%$ of DNA GRT, lamivudine and emtricitabine in $6.3 \%$, efavirenz and nevirapine in $5.3 \%$, and elvitegravir in $3.9 \%$. $\mathrm{M} 230 \mathrm{I}$ and E138K were the two most common rilpivirine resistance-associated mutations discovered in DNA GRT, in, respectively, $9.9 \%$ and $2.6 \%$ of the tests. M184I and M184V were the two most frequent $3 \mathrm{TC}$ resistance-associated mu- tations newly diagnosed with DNA GRT, with, respectively, $5.6 \%$ and $2.0 \%$ of the tests.

In case of DNA GRT performed in a context of a virological success, new resistances were more frequently detected (33\% vs. $24 \%, p=0.09)$, while previously known resistances were less frequently detected (34\% vs. $45 \%$, $p=0.04)$.

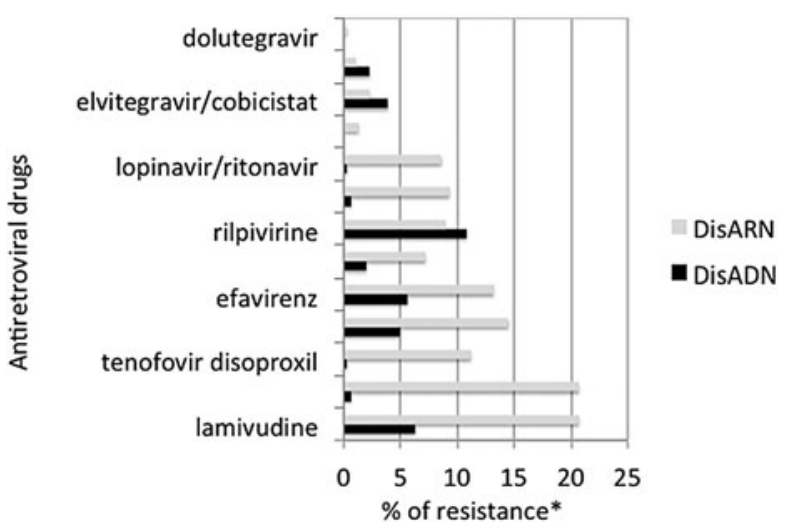

FIG. 1. Discordance between DNA genotype and historical RNA. *Resistance was defined as the presence of at least one mutation inducing possible or full resistance to a drug according to the ANRS algorithm. Results are expressed by $\%$ of DNA GRTs revealing a resistance out of the 304 tests included in the analysis. DisDNA, resistance diagnosed by DNA GRT, but never detected in RNA; DisRNA, resistance absent from DNA GRT, but detected at least once in RNA. ANRS, Agence Nationale de Recherche sur le SIDA; GRT, genotypic resistance test. 
Table 2. Change of Antiretroviral Regimen Following DNA Genotypic Resistance Test, Depending on the Virological Status

\begin{tabular}{|c|c|c|c|c|}
\hline ART changes & $\begin{array}{c}\text { Total }(\mathrm{n}=104) \\
\mathrm{n}(\%)\end{array}$ & $\begin{array}{c}V L>20 \text { copies } / m L \\
(\mathrm{n}=31) \mathrm{n}(\%)\end{array}$ & $\begin{array}{c}V L<20 \text { copies } / m L \\
(\mathrm{n}=73) \mathrm{n}(\%)\end{array}$ & $\mathrm{p}$ \\
\hline INSTI_-based regimen & $62(60)$ & $11(35)$ & $51(70)$ & 0.002 \\
\hline Rilpivirine containing regimen & $7(7)$ & $1(3)$ & $6(8)$ & 0.62 \\
\hline STR & $35(34)$ & $5(16)$ & $30(41)$ & 0.02 \\
\hline Deintensification & $22(21)$ & $8(26)$ & 14 (19) & 0.41 \\
\hline Change of third agent & $17(16)$ & $4(13)$ & $13(18)$ & 0.74 \\
\hline Change of NRTI & $6(6)$ & $1(3)$ & $5(7)$ & 0.79 \\
\hline Intensification & $4(4)$ & $2(6)$ & $2(3)$ & 0.73 \\
\hline Others & $20(19)$ & $11(35)$ & $9(12)$ & 0.014 \\
\hline
\end{tabular}

ART, antiretroviral therapy; INSTI, integrase strand transfer inhibitor; NRTI, nucleoside reverse transcriptor inhibitor; STR, single-tablet regimen; VL, viral load.

Significant results $(p<0.05)$ appear in bold.

\section{Antiretroviral therapy (ART) change}

A change of ART was performed after 104 (34\%) DNA GRT. ART changes according to the virological status at the time of DNA GRT performance are summarized in Table 2. They were mainly switch for a single-tablet regimen $(34 \%)$, or a deintensification strategy $(21 \%)$. Changes were more frequent in the context of virological success (39\% vs. $26 \%$, $p=0.02$ ). An integrase strand transfer inhibitor (INSTI) was prescribed in $60 \%$ of patients.

Factors associated with decision to modify ART in case of virological success were higher age $(51 \pm 11$ vs. $47 \pm 11$ years, $p<0.001)$ and longer time with $\mathrm{VL}<20$ copies/mL $(1352 \pm 171$ vs. $1057 \pm 106$ days, $p<0.001)$. In the context of low-level viremia, detection of a new resistance on DNA GRT was associated with ART change (48\% vs. $17 \%, p=0.001$ ).

\section{Virological outcome}

In situation of virological success, proportion of viremia occurrence after 6 months of follow-up was lower after ART change guided by DNA GRT in addition to analysis of historical RNA GRTs than after continuation of previous ART $(5 \%$ vs. $19 \%, p=0.02)$. Figure $2 \mathrm{~A}$ shows Kaplan-Meier estimates of cumulative probability of maintenance of virological success after choice of ART guided by DNA GRT. Probability of maintenance of virological success was significantly higher after ART change (Log-rank, $p=0.006)$. In multivariate analysis (Table 3), the only factor independently associated with occurrence of viremia was higher HIV DNA quantification $(p=0.01)$. On the opposite, a higher nadir of CD4 count $(p=0.04)$ and a longer time with CV <20 copies/mL $(p=0.04)$ were independently associated with a lower risk of viremia occurrence.

In situation of low-level viremia, choice of ART guided by DNA GRT in addition to analysis of historical RNA GRTs led to virological success in $68 \%$ of cases. In this last situation, virological success was obtained after ART change guided by DNA GRT in $52 \%$, while decision to maintain same ART led to virological success in $74 \%(p=0.04)$. Figure 2B shows Kaplan-Meier estimates of cumulative probability of persistence of virological failure after choice of ART guided by DNA GRT. Probability of persistence of virological failure was significantly lower in absence of ART change (Log-rank, $p<0.001$ ). In absence of ART change, success occurred usually soon after DNA GRT performance. In multivariate analysis (Table 3), the only factor independently associated with persistence of virological replication was longer time with VL $>20$ copies/mL $(p=0.02)$.
A

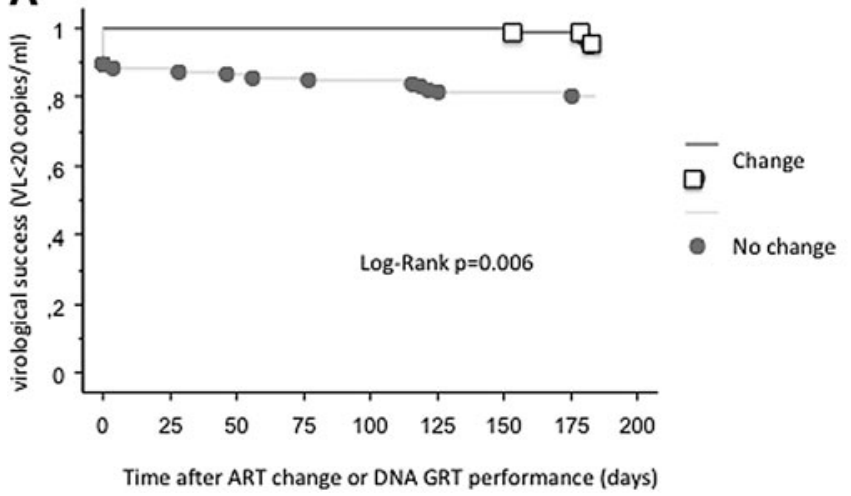

B

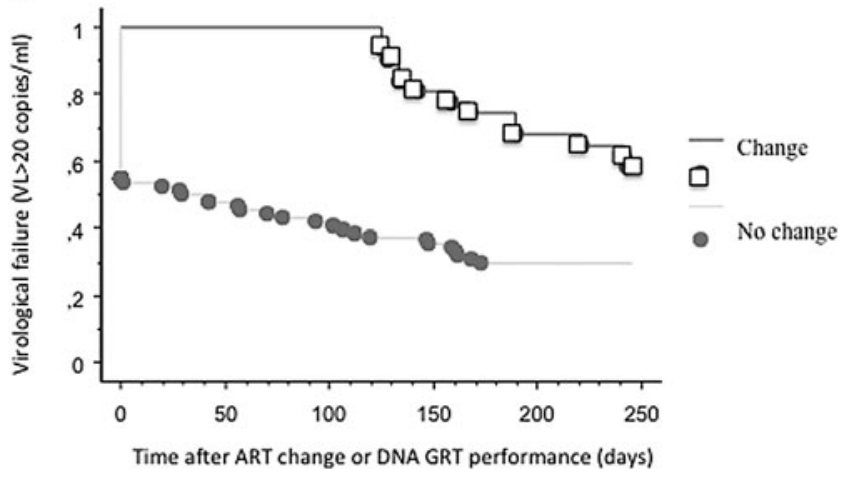

FIG. 2. (A) Kaplan-Meier estimates of cumulative probability of maintenance of virological success after choice of ART guided by DNA GRT in addition to historical RNA GRTs. (B) Kaplan-Meier estimates of cumulative probability of persistence of virological failure despite choice of ART guided by DNA GRT in addition to historical RNA GRTs. ART, antiretroviral therapy; GRT, genotypic resistance test. 
Table 3. Factors Independently Associated with Virological Outcome

\begin{tabular}{|c|c|c|c|c|}
\hline \multirow[b]{2}{*}{ Characteristics at the time of DNA GRT performance } & \multicolumn{2}{|c|}{ Univariate analysis } & \multicolumn{2}{|c|}{ Multivariate analysis } \\
\hline & $O R(95 \% C I)$ & $\mathrm{p}$ & OR $(95 \% C I)$ & $\mathrm{p}$ \\
\hline \multicolumn{5}{|c|}{ Factors associated with occurrence of virological failure in situation of virological success } \\
\hline Sex (male) & $1.17(0.46-2,97)$ & 0.74 & & \\
\hline CDC stage (stage C) & $1.19(0.5-2.84)$ & 0.70 & & \\
\hline Non-B HIV subtypes & $2.6(1.11-6.29)$ & 0.03 & $1.67(0.50-5.58)$ & 0.41 \\
\hline Nadir of CD4 count (per 50 cells $/ \mathrm{mm}^{3}$ ) & $0.99(0.94-1.01)$ & 0.09 & $0.74(0.55-0.99)$ & 0.04 \\
\hline Zenith VL (>100,000 copies/mL) & $4.26(1.8-10.08)$ & $<0.001$ & $0.88(0.24-3.21)$ & 0.84 \\
\hline CD4 count $\left(>500 / \mathrm{mm}^{3}\right)^{1}$ & $0.65(0.28-1.54)$ & 0.33 & & \\
\hline HIV DNA quantification (per $10010^{6} \mathrm{PBMC}$ ) & $1.10(1.02-1.20)$ & 0.003 & $1.11(1.01-1.22)$ & 0.01 \\
\hline Time with VL $<20$ copies/mL (per 28 days) & $0.97(0.94-0.99)$ & 0.002 & $0.97(0.95-1.00)$ & 0.04 \\
\hline Time between ART initiation and DNA GRT $(<1$ year) & $0.79(0.09-7.01)$ & 0.83 & & \\
\hline Time between last ART change and DNA (<1 year) & $1.34(0.43-4.16)$ & 0.61 & & \\
\hline DisDNA & $0.75(0.30-1.90)$ & 0.55 & & \\
\hline \multicolumn{5}{|c|}{ Factors associated with occurence of virological success in situation of low-level viremia } \\
\hline Sex (male) & $1.34(0.48-3.72)$ & 0.58 & & \\
\hline CDC stage (stage C) & $1.19(0.5-2.84)$ & 0.70 & & \\
\hline Non-B HIV subtypes & $1.45(0.62-3.42)$ & 0.39 & & \\
\hline Nadir of CD4 count (per 50 cells $/ \mathrm{mm}^{3}$ ) & $0.73(0.3-1.76)$ & 0.48 & & \\
\hline Zenith VL (<100,000 copies/mL) & $2.8(1.18-6.67)$ & 0.02 & $0.61(0.21-1.72)$ & 0.35 \\
\hline CD4 count $\left(>500 / \mathrm{mm}^{3}\right)$ & $0.92(0.39-2.15)$ & 0.85 & & \\
\hline HIV DNA quantification (per $10010^{6} \mathrm{PBMC}$ ) & $1(0.999-1.00)$ & 0.48 & & \\
\hline Time with VL $>20$ copies/mL (per 28 days) & $0.98(0.97-1.00)$ & 0.11 & $0.97(0.97-0.99)$ & 0.02 \\
\hline Time between last ART change and DNA $(<1$ year $)$ & $0.34(0.06-1.76)$ & 0.20 & $0.15(0.01-2.27)$ & 0.17 \\
\hline DisDNA & $0.96(0.36-2.60)$ & 0.94 & & \\
\hline
\end{tabular}

DisDNA, resistance diagnosed by DNA GRT but never detected in RNA.

ART, antiretroviral therapy; CI, confidence interval; DNA GRT, DNA genotypic resistance test; OR, odds ratio; PBMC, peripheral blood mononuclear cell; VL, viral load.

Significant results $(p<0.05)$ appear in bold.

\section{Discussion}

In our cohort, we identified two distinct populations who beneficed from the realization of DNA GRT: patients experimenting successful virological suppression and patients with low-level viremia. Virologically suppressed patients were more numerous, reflecting the improvement in ART leading to high rates of virological success. But new concerns have risen among clinicians, including long-term tolerability of ART and improvement of patients' life quality.

In France, recent guidelines proposed to realize proviral DNA GRT not only in patients with virological failure with low-level viremia and unsuccessful RNA resistance test but also in patients successfully suppressed when no historical RNA resistance test is available and a switch of therapy is planned.

In our cohort, majority of DNA GRT performed routinely in the context of detectable VL was made in a context of low-level viremia (VL <200 copies/mL). Indeed, 11 DNA GRTs were performed while HIV-VL was higher than 1000 copies $/ \mathrm{mL}$ and RNA GRT could have been performed successfully. Only 23 DNA tests were performed in patients experimenting virological failure and low-level viremia (VL between 200 and 500 copies/mL), as proposed in the French guidelines. Recently, genotypic resistance tests using additional nested polymerase chain reaction showed high success rate even at low copies of HIV-RNA. ${ }^{15}$ Success rate of amplification and sequencing dropped only for viremia levels below 200 copies $/ \mathrm{mL}$. We choose to retain in our analysis patients with low-level viremia (<200 copies/mL), who represented the majority of patients with DNA GRT performed with a detectable VL and for whom RNA GRT is generally unsuccessful.
In case of virological success, DNA GRT is recommended in the absence of at least one historical RNA GRT. Prior RNA GRT was available in almost two thirds of our patients. In our cohort, in this context, mean time between last RNA GRT and DNA GRT was 6 years. The majority of previous RNA GRT has been performed at a time when specific mutations concerning rilpivirine were not searched and integrase sequencing was not performed. Rilpivirine resistance-associated mutations were first described by analyzing the HIV strains from patients experiencing virological failure under rilpivirine therapy. ${ }^{16,17}$

Several studies have demonstrated the risk of archived resistance to rilpivirine especially in patients who had experienced previous virological failure while receiving non-nucleoside reverse transcriptase inhibitor (NNRTI). ${ }^{18-21}$ Lambert-Niclot et al. found good concordance between DNA and RNA GRT only in absence of prior virological failure. ${ }^{11}$ DNA GRT could be useful in a virologically suppressed patient in the absence of prior RNA GRT, or in case of history of virological failure while receiving NNRTI and no RNA GRT at the time of failure. Use of rilpivirine should be avoided if resistance-associated mutations are detected using a DNA GRT. On the opposite, pretreatment assessment for INSTI resistance is not widely recommended since prevalence of resistance is low among naive patients. ${ }^{22,23}$ However, for patients who failed a raltegravir containing regimen, who are currently virologically suppressed, and were not tested for resistance at failure, DNA GRT could also be useful to identify archived resistances. ${ }^{12}$

In our cohort, more than one out of four DNA GRTs revealed at least one resistance not detected in any previous RNA GRTs. Resistances detected only in DNA GRT concerned mainly NNRTI, especially rilpivirine in $10 \%$ of cases. 
But resistances to integrase inhibitors were also identified in $4 \%$ of cases. Zaccarelli et al. found similar results in a population of drug-experienced patients with suppressed or lowlevel viremia. ${ }^{13}$ DNA GRTs might provide useful information in addition with historical RNA GRTs, especially in patients highly ART experimented. ${ }^{24}$

In our cohort, results of proviral DNA GRT in addition to analysis of historical RNA GRTs led to ART change in one third of cases. DNA GRTs performed in case of virological success were more often followed by ART change, especially in older patients experimenting longer viral suppression. Mean duration of viral suppression $(\mathrm{CV}<20$ copies/mL) was 3.7 years in virologically suppressed patients changing ART. Various switching strategies have been used in the modern era of antiretroviral therapy to avoid adverse effects, enhance adherence, and improve life-quality. ${ }^{25}$ Inclusion criteria in those clinical trials usually specified a minimum duration of virological suppression of 6 months. ${ }^{26-28}$ Similarly studies evaluating maintenance strategies with dual therapy required at least 6 months of viral suppression. ${ }^{29,30}$ But longer duration (as long as 2 years) was sometimes preferred. ${ }^{31}$

In our cohort, under these circumstances, change of ART appeared safe with subsequent virological failure in only $5 \%$ of cases. Factors independently associated with occurrence of virological failure were higher HIV DNA quantification, lower nadir of CD4 count, and a shorter time with $\mathrm{CV}<20$ copies/mL. Similarly, in the only previously published study assessing the role of resistance in PBMC GRT on predicting virological outcome in virologically suppressed patients, Armenia et al. found a significant impact of the time of previous virological suppression and of nadir of CD4 cell count on virological outcome after switching ART. ${ }^{32}$

These results were in accordance with predictive factors of virological response in deintensification studies. ${ }^{33,34} \mathrm{~A}$ recent trial of deintensification retained HIV DNA quantification as an inclusion criteria, with a threshold of $<2.7 \log _{10}$ copies $/ 10^{6}$ PBMCs. ${ }^{35}$ In our cohort, no impact on virological outcome was found of detection of new resistance on DNA GRT in patients virologically suppressed. In their study assessing the role of resistance in PBMC GRT, Armenia et al. found a higher risk of virological failure in patients switching therapy while infected with a virus not fully susceptible according to DNA GRT. ${ }^{32}$

In our cohort, results of DNA GRT were obtained before decision to change or not ART. Clinicians took into account DNA GRT results in addition to previous plasma resistance history and previous viroimmunological history, for the choice of subsequent treatment. Even if some of the detected mutations could be harbored only by defective variants. ${ }^{36}$ Indeed, the most frequent resistance-associated mutations detected in the DNA GRT, but not in the RNA genotype, were M230I, M184I, and E138K. Several studies have detected the presence of these mutations as a result of APOBEC $3 \mathrm{G} / \mathrm{F}$ hypermutation on HIV genome. ${ }^{37,38}$ APOBEC $3 \mathrm{G} / \mathrm{F}$ activity generates replicationincompetent viruses. Drug-resistant variants could be defective and have no significant impact on virological outcome.

In case of DNA GRT performed in patients with persistent low-level viremia, the detection of a new resistance on DNA GRT was associated with ART change. In this situation of low-level viremia, choice to modify or not ART, guided by DNA GRT in addition to analysis of previous RNA GRTs and therapeutic and immunovirological history led to virological success in almost two thirds of cases.
Virological success was obtained less frequently after ART change despite the consideration of RNA historical resistanceassociated mutation. In our cohort, a new measure of VL was often performed soon after low-level viremia appearance and DNA GRT performance to confirm viral replication. ART change was decided only in absence of spontaneous virological control, explaining partially why success was more frequent in patients maintaining same ART. Performance of DNA GRT could have been avoided in the cases of rapid virological control, but predictive factors of viral replication persistence are difficult to identify. In our cohort, the only factor independently associated with persistence of virological replication was longer prior duration of $\mathrm{VL}>20$ copies $/ \mathrm{mL}$.

Our results confirmed previous data that suggests an association of persistent low-level viremia with deleterious virological outcome. Falasca et al. identified higher values of viremia copy-years, defined as the area under plasma VL curve as a risk factor of virological failure in long-termtreated patients. ${ }^{39}$ Similarly a persistent low-level viremia, defined as a VL of 50-199 copies/mL on at least $25 \%$ of measurements was associated with virological failure. ${ }^{40}$ But clear cutoffs are lacking to allow identification of patients at high risk of virological failure. In our cohort, numerous patients experienced virological control soon after DNA GRT performance in the absence of any ART change. Having better predictors of virological failure could help select more effectively the patients with low-level viremia who will benefit from DNA GRT.

Our study has several limits. Because of the retrospective nature of our study, patients who have benefited from a DNA GRT were selected by the prescribers as being the most likely to take advantage of the results of this test. But other patients could have been good candidates for DNA GRT performance. Moreover, it is a monocentric study with a limited number of prescribers. Our findings might not be generalized as clinical practice may differ across institutions and countries.

In conclusion, use of HIV-1 proviral DNA GRT to guide ART optimization in situation of virological success seems safe with a low rate of virological failure at 6 months. In situation of persistent low-level viremia, choice guided by DNA GRT to modify or not ART led to virological success in almost two thirds of cases. Our results suggest that DNA GRT can be a useful tool to choose subsequent ART in both virologically suppressed patients and patients experimenting lowlevel virological replication in addition to analysis of previous cumulative resistance and viroimmunological history.

\section{Author Disclosure Statement}

No competing financial interests exist.

\section{Funding Information}

No funding was received for this article.

\section{References}

1. Vandamme AM, Camacho RJ, Ceccherini-Silberstein F, et al. European recommendations for the clinical use of HIV drug resistance testing: 2011 update. AIDS Rev 2011; 13:77-108.

2. French guidelines for the medical management of people living with HIV. Experts group Recommendations, directed by Pr Morlat, under the control of CNS and ANRS. November 
2016. Available at: https://cns.sante.fr (Last accessed January 2020).

3. Li JZ, Gallien S, Do TD, et al. Prevalence and significance of HIV-1 drug resistance mutations among patients on antiretroviral therapy with detectable low-level viremia. Antimicrob Agents Chemother 2012;56:5998-6000.

4. Lambotte O, Chaix ML, Gubler B, et al. The lymphocyte HIV reservoir in patients on long-term HAART is a memory of virus evolution. AIDS 2004;18:1147-1158.

5. Verhofstede C, Noe A, Demecheleer E, et al. Drug-resistant variants that evolve during non suppressive therapy persist in HIV-1-infected peripheral blood mononuclear cells after long-term highly active antiretroviral therapy. J Acquir Immune Defic Syndr 2004;35:473-483.

6. Turriziani O, Andreoni M, Antonelli G. Resistant viral variants in cellular reservoirs of human immunodeficiency virus infection. Clin Microbiol Infect 2010;16:1518-1524.

7. Diaz RS, Accetturi CA, Sucupira MC. Emergence of resistance mutations preceding virologic failure in patients receiving antiretroviral therapy. J Acquir Immune Defic Syndr 2008;49:111-113.

8. Palmisano L, Galluzzo CM, Giuliano M. The importance of testing genotypic resistance in proviral DNA of patients fully responding to highly active antiretroviral therapy. J Acquir Immune Defic Syndr 2009;51:233-234.

9. Delaugerre C, Braun J, Charreau I, et al. Comparison of resistance mutation patterns in historical plasma HIV RNA genotypes with those in current proviral HIV DNA genotypes among extensively treated patients with suppressed replication. HIV Med 2012;13:517-525.

10. Wirden M, Soulie C, Valantin MA, et al. Historical HIVRNA resistance test results are more informative than proviral DNA genotyping in case of suppressed or residual viraemia. J Antimicrob Chemother 2011;66:709-712.

11. Lambert-Niclot S, Grude CA, Flandre P, et al. Usefulness of an HIV DNA resistance genotypic test in patients who are candidates for a switch to the rilpivirine/emtricitabine/ tenofovir disoproxil fumarate combination. J Antimicrob Chemother 2016;71:2248-2251.

12. Fernandez-Caballero JA, Chueca N, Alvarez M, et al. Usefulness of integrase resistance testing in proviral HIV-1 DNA in patients with raltegravir prior failure. BMC Infect Dis 2016;16:197.

13. Zaccarelli M, Santoro MM, Armenia D, et al. Genotypic resistance test in proviral DNA can identify resistance mutations never detected in historical genotypic test in patients with low level or undetectable HIV-RNA. J Clin Virol 2016;82:94-100.

14. Pugliese P, Cuzin L, Enel P, et al. NADIS 2000, development of an electronic medical record for patients infected by HIV, HBV and HCV. Presse Med 2003;32:299-303.

15. Santoro MM, Fabeni L, Armenia D, et al. Reliability and clinical relevance of the HIV-1 drug resistance test in patients with low viremia. Clin Infect Dis 2014;58:1156-1164.

16. Rimsky L, Vingerhoets J, Van Eygen V, et al. Genotypic and phenotypic characterization of HIV-1 isolates obtained from patients on rilpivirine therapy experiencing virologic failure in the phase $3 \mathrm{ECHO}$ and THRIVE studies: 48-week analysis. J Acquir Immune Defic Syndr 2012;59:39-46.

17. Rimsky L, Van EygenV, Hoogstoel A, et al. 96-week resistance analyses of rilpivirine in treatment-naïve, HIV-1infected adults from the ECHO and THRIVE Phase III trials. Antivir Ther 2013;18:967-977.
18. Gallien S, Charreau I, Nere ML, et al. Archived HIV1DNA resistance mutations to rilpivirine and etravirine in successfully treated HIV-1-infected individuals preexposed to efavirenz or nevirapine. J Antimicrob Chemother 2015;70:562-565.

19. Anta L, Llibre JM, Poveda E, et al. Rilpivirine resistance mutations in HIV patients failing non-nucleoside reverse transcriptase inhibitor-based therapies. AIDS 2013;27: 81-85.

20. Lambert-Niclot S, Charpentier C, Storto A, et al. Rilpivirine, emtricitabine and tenofovir resistance in HIV-1infected rilpivirine-naive patients failing antiretroviral therapy. J Antimicrob Chemother 2014;69:1086-1089.

21. Jeulin H, Foissac M, Boyer L, et al. Real-life rilpivirine resistance and potential emergence of an E138A-positive HIV strain in north-eastern France. J Antimicrob Chemother 2014;69:3095-3102.

22. Koullias Y, Sax PE, Fields NF, et al. Should we be testing for baseline integrase resistance in patients newly diagnosed with human immunodeficiency virus? Clin Infect Dis 2017;65:1274-1281.

23. Ambrosioni J, Mosquera M, Miro JM. Resistance testing for integrase-strand transfer inhibitors in naive HIVinfected individuals. Clin Infect Dis 2019;68:1976-1977.

24. Kabamba-Mukadi B, Duquenne A, Henrivaux P, et al. HIV-1 proviral resistance mutations: Usefulness in clinical practice. HIV Med 2010;11:483-492.

25. Prieto P, Podzamczer D. Switching strategies in the recent era of antiretroviral therapy. Expert Rev Clin Pharmacol 2019;12:235-247.

26. Palella FJ, Fisher M, Tebas $\mathrm{P}$, et al. Simplification to rilpivirine/emtricitabine/tenofovir disoproxil fumarate from ritonavir-boosted protease inhibitor antiretroviral therapy in a randomized trial of HIV-1 RNA-suppressed participants. AIDS 2014;28:335-344.

27. Arribas JR, Pialoux G, Gathe J, et al. Simplification to coformulated elvitegravir, cobicistat, emtricitabine, and tenofovir versus continuation of ritonavir-boosted protease inhibitor with emtricitabine and tenofovir in adults with virologically suppressed HIV (STRATEGY-PI): 48 week results of a randomised, open-label, phase $3 \mathrm{~b}$, noninferiority trial. Lancet Infect Dis 2014;14:581-589.

28. Trottier B, Lake JE, Logue K, et al. Dolutegravir/abacavir/lamivudine versus current ART in virally suppressed patients (STRIIVING): A 48-week, randomized, non-inferiority, openlabel, Phase IIIb study. Antivir Ther 2017;22:295-305.

29. Perez-Molina JA, Rubio R, Rivero A, et al. Dual treatment with atazanavir-ritonavir plus lamivudine versus triple treatment with atazanavir-ritonavir plus two nucleos(t)ides in virologically stable patients with HIV-1 (SALT): 48 week results from a randomised, open-label, non-inferiority trial. Lancet 2015;15:775-784.

30. Llibre JM, Hung CC, Brinson C, et al. Efficacy, safety, and tolerability of dolutegravir-rilpivirine for the maintenance of virological suppression in adults with HIV-1: Phase 3, randomised, non-inferiority SWORD-1 and SWORD-2 studies. Lancet 2018;391:839-849.

31. Joly V, Burdet C, Landman R, et al. Dolutegravir and lamivudine maintenance therapy in HIV-1 virologically suppressed patients: Results of the ANRS 167 trial (LAMIDOL). J Antimicrob Chemother 2019;74:739-745.

32. Armenia D, Zaccarelli M, Borghi V, et al. Resistance detected in PBMCs predicts virological rebound in HIV-1 
suppressed patients switching treatment. J Clin Virol 2018; 104:61-64.

33. Lambert-Niclot S, Flandre P, Valantin MA, et al. Factors associated with virological failure in HIV-1-infected patients receiving darunavir/ritonavir monotherapy. J Infect Dis 2011;204:1211-1216.

34. Wijting IEA, Rutsaert SL, Rokx C, et al. Predictors of virological failure in HIV-1-infected patients switching to dolugravir maintenance monotherapy. HIV Medicine 2019; 20:63-68.

35. Prazuck T, Verdon R, Le Moal G, et al. Trial to evaluate the interest of a reductive antiretroviral strategy using oral therapy in spite of triple therapy (TRULIGHT): Results at W48. Med Mal Infect 2019;S21-S23.

36. Alidjinou EK, Deldalle J, Robineau O, et al. Routine drug resistance testing in proviral HIV-1 DNA: Prevalence of stop codons and hypermutation and associated factors. J Med Virol 2019;91:1684-1687.

37. Boukli N, Boyd A, Collot M, et al. Utility of HIV-1 DNA genotype in determining antiretroviral resistance in patients with low or undetectable HIV RNA viral loads. J Antimicrob Chemother 2018;73:3129-3136.
38. Noguera-Julian M, Cozzi-Lepri A, Di Giallonardo F. Contribution of APOBEC3G/F activity to the development of low-abundance drug-resistant human immunodeficiency virus type I variants. Clin Microbiol Infect 2016;22:191200.

39. Falasca F, De Vito C, Mazzuti L, et al. Copy-years viremia and risk of virological failure in long-term-treated HIV patients. J Acquir Immune Defic Syndr 2019;80:423-438.

40. Joya C, Won SH, Schofield C, et al. Persistent low-level viremia while on antiretroviral therapy is an independent risk factor for virologic failure. Clin Infect Dis 2019;69: 2145-2152.

Address correspondence to:

Agnès Meybeck, MD

Infectious Diseases Department

Tourcoing Hospital

135 Avenue du Président Coty

Tourcoing 59200

France

E-mail: agnesmeybeck@yahoo.fr 